\title{
A toxidez de diversas lantanas para bovinos e ovinos no Brasil $^{1}$
}

\author{
Marilene de Farias Brito² ${ }^{2}$ Carlos Hubinger Tokarnia ${ }^{3 *}$ e Jürgen Döbereiner ${ }^{4}$
}

\begin{abstract}
Brito M.F., Tokarnia C.H. \& Döbereiner J. 2004. [The toxicity of diverse lantanas for cattle and sheep in Brazil.] A toxidez de diversas lantanas para bovinos e ovinos no Brasil. Pesquisa Veterinária Brasileira 24(3):153-159. Projeto Sanidade Animal Embrapa/UFRRJ, Km 47, Seropédica, RJ 23890-000, Brazil. E-mail: tokarnia@ufrrj.br

Experiments on the toxicity of Lantana spp for cattle and sheep revealed a similar situation as is reported from Australia. Neither all Lantana species nor all varieties which occur in Brazil are poisonous. Lantana samples collected at Boa Vista (Roraima), Castanhal (Pará), Cáceres (Mato Grosso), Serra Talhada (Pernambuco), Cabo Frio and Quatis (Rio de Janeiro), and Canoinhas (Santa Catarina) were toxic. All the other Lantana samples, collected at Cruzeiro do Sul (Acre), Castanhal (Pará), Chapada dos Guimarães and Lambari d'Oeste (Mato Grosso), Jaguaribe (Ceará), Vitória da Conquista and Wanderley (Bahia), Vitória (Espírito Santo), Vassouras, Parati and Itaguaí (Rio de Janeiro) revealed experimentally as non-toxic at a dose of $40 \mathrm{~g} / \mathrm{kg}$. In all cases the lethal dose was $40 \mathrm{~g} / \mathrm{kg}$, with two exceptions: the sample collected at Canoinha (Santa Catarina) was much more toxic $(10 \mathrm{~g} / \mathrm{kg})$ and the one from Serra Talhada (Pernambuco) was much less toxic, as this last sample caused lethal poisoning only at a dosage of $40 \mathrm{~g} / \mathrm{kg} /$ day given during 30 days. Histories about the observation of photosensitization in cattle due to the ingestion of Lantana spp were obtained at Serra Talhada (Pernambuco), Cáceres (Mato Grosso), Cabo Frio and Quatis (Rio de Janeiro), and Canoinhas (Santa Catarina). No correlation could be established between the colour of the flowers of Lantana spp and the toxicity of the plants. This confirms reports from Australia that the toxic potential of the Lantana species is not necessarily related to the colour of their flowers.
\end{abstract}

INDEX TERMS: Poisonous plants, plant poisoning, Lantana spp, Verbenaceae, sheep, cattle, pathology.

RESUMO.- Neste estudo sobre a toxidez das lantanas para bovinos e ovinos, verificou-se situação semelhante à que ocorre na Austrália, que nem todas as espécies de Lantana e nem todos os taxa de Lantana camara que ocorrem no Brasil, são tóxicos. Verificou-se, que foram tóxicas as amostras de lantanas procedentes de Boa Vista (RO), Castanhal (PA), Cáceres (MT), Serra Talhada $(\mathrm{PE})$, Cabo Frio e Quatis (RJ) e Canoinhas (SC). Outras amostras procedentes de Cruzeiro do Sul (Acre), Castanhal (PA), Chapada

\footnotetext{
${ }^{1}$ Recebido em 19 de abril de 2004.

Aceito para publicação em 15 de junho de 2004.

2 Depto Epidemiologia e Saúde Pública, Setor de Anatomia Patológica, Universidade Federal Rural do Rio de Janeiro (UFRRJ), Km 47, Seropédica, RJ 23890-000.

${ }^{3}$ Depto Nutrição Animal e Pastagem, Inst. Zootecnia, UFRRJ, Km 47, Seropédica, RJ 23835-000. *Autor para correspondência. E-mail: tokarnia@ufrrj.br

${ }^{4}$ Projeto Sanidade Animal Embrapa/UFRRJ, Km 47, Seropédica, RJ 23890-000. Bolsista do CNPq (Proc.305294/1988-1).
}

dos Guimarães e Lambari d' Oeste (MT), Jaguaribe (CE), Vitória da Conquista e Wanderley (BA), Vitória (ES), Vassouras, Parati e Itaguaí (RJ) não revelaram toxidez nas doses administradas (40 $\mathrm{g} / \mathrm{kg}$ ). A dose letal das amostras submetidas à experimentação no Brasil foi bastante constante $(40 \mathrm{~g} / \mathrm{kg}$ ), com duas exceções: as folhas frescas da lantana procedente de Canoinha (SC) foram muito mais tóxicas $(10 \mathrm{~g} / \mathrm{kg}$ ) e as folhas frescas e as dessecadas da lantana de Serra Talhada (PE) foram muito menos tóxicas, pois somente causaram intoxicação não-letal com a dose de $40 \mathrm{~g} /$ $\mathrm{kg} /$ dia administradas durante 30 dias. Históricos so bre a observação de fotossensibilização em bovinos, ligados à ingestão de lantanas foram obtidos em Serra Talhada (PE), Cáceres (MT), Cabo Frio (RJ), Quatis (RJ) e Canoinhas (SC). Verificou-se que não é possível estabelecer uma correlação entre a cor das inflorescências das lantanas e sua toxidez, confirmando a constatação feita na Austrália, que o potencial de intoxicar não está necessariamente relacionada com a cor das flores.

TERMOS DE INDEXAÇÃO: Plantas tóxicas, intoxicação por planta, Lantana spp, Verbenaceae, ovinos, bovinos, patologia. 


\section{INTRODUÇÃO}

São conhecidas mais que 50 espécies do gênero Lantana, pertencente à família Verbenaceae. Lantana spp são plantas arbustivas conhecidas pelos nomes populares de "chumbinho", "camará", "cambará", "bem-me-quer" e "mal-me-quer".

A literatura so bre a toxidez das plantas desse gênero se refere, sobretudo, a Lantana camara L. e suas variedades, mas são citadas também algumas outras espécies como tóxicas. Existem muitos taxa (variedades) de L. camara; eles variam na cor das flores, no habitat e numa série de características morfológicas (Harley 1973). É importante considerar que nem todas as espécies de Lantana e nem todos os taxa de L. camara são tóxicos. A capacidade de intoxicar não está necessariamente relacionada à cor das flores (Seawright 1963).

Trata-se de plantas cosmopolitas. Acredita-se que L. camara e a maioria das outras espécies desse gênero são originárias das partes tropical e subtropical do Continente Americano; elas teriam sido levadas, como plantas ornamentais, para outros países com climas semelhantes, onde se difundiram (Seawright 1963, 1965b, Aluja 1971).

Na Austrália, além de ser considerada uma das plantas tóxicas mais importantes, especialmente na costa de Queensland, onde causa a morte de 1000 a 1500 bovinos por ano, L. camara é importante também como planta invasora; nesse continente a planta encontrou ambiente tão favorável que cobriu extensas áreas, tornando-as não-utilizáveis pelo homem (Seawright 1965b, Harley 1973).

No Brasil Lantana spp são encontradas desde a Amazônia até o Rio Grande do Sul, em agrupamentos maiores ou menores, invadindo áreas de pastagens nativas ou cultivadas, mas não dominando a vegetação como no caso da Austrália.

Sob condições naturais, a intoxicação por Lantana spp tem sido descrita em bovinos, tanto no Brasil (Tokarnia et al. 1984, Riet-Correa et al. 1984), como em outros países (Turbet 1928, 1931, Sanders 1946, Brooks 1961, Seawright 1963, Hall 1964, Aluja 1970, Seawright \& Allen 1972, Yadava \& Verma 1978, Furie et al. 1987).

Pass (1986) informa que, na Austrália, os bovinos são mais afetados por Lantana spp. do que outras espécies animais, mas que a doença ocorreria também em ovinos e caprinos.

Com base em observações obtidas no Brasil (Tokarnia et al. 1984, Riet-Correa et al. 1984) e nos dados fornecidos pela literatura de outros países (Turbet 1928, 1931, Sanders 1946, Brooks 1961, Seawright 1963, Hall 1964, Aluja 1970, Yadava \& Verma 1978), pode-se deduzir que, em geral, são necessários vários fatores para que ocorra a intoxicação natural por Lantana spp; que os animais estejam com fome, que sejam transferidos de pasto ou região, que haja espécie ou variedade tóxica de Lantana e que a planta exista em quantidade suficiente no local. Desta maneira a intoxicação por Lantana não é comum, mas quando ocorre, tem alto índice de morbidade e letalidade.

A intoxicação experimental por Lantana spp tem sido reproduzida, no Brasil, em bovinos (Silva \& Couto 1971, Tokarnia et al. 1984, 1999, Riet-Correa et al. 1984), ovinos (Brito \& Tokarnia 1995), búfalos (Láu 1990) e coelhos (Brito 1995). Em outros países a intoxicação experimental foi realizada, em bovinos
(Turbet 1928, Aluja 1970, Seawright \& Allen 1972, Aguilera et al. 1986, Furie et al. 1987), búfalos (Kalra et al. 1984), ovinos (Steyn \& van der Walt 1941, Seawright 1963, 1964, 1965c, Gopinath \& Ford 1969, Aluja 1971, Pass et al. 1978), caprinos (Obwolo et al. 1990, 1991) e coelhos (Youssef et al. 1984, Sharma et al. 1988); em cobaias, a reprodução foi feita com extrato de L. camara por via oral (Seawright 1965d).

São tóxicas tanto as folhas frescas quanto as dessecadas. A planta não perde em toxidez durante o processo de dessecagem e a mantém durante pelo menos um ano (Brito \& Tokarnia 1995).

No Brasil, as quantidades necessárias para causar intoxicação com Lantana spp em bovinos e ovinos foram verificadas em alguns estudos experimentais. Assim, Silva \& Couto (1971) administraram Lantana camara (coletada em Pernambuco) em estado fresco ou dessecado, por via oral, a oito bovinos, nas dosagens de 10 a $40 \mathrm{~g} / \mathrm{kg} / \mathrm{dia}$ durante 15 a 30 dias, tendo observado leves sintomas de fotossensibilização nos bovinos que ingeriram a planta fresca ou dessecada nas doses diárias de 40 e $20 \mathrm{~g} / \mathrm{kg}$ durante 30 dias.

Tokarnia et al. (1984) administraram Lantana tiliaefolia Cham. (coletada em Mato Grosso, mun. Cáceres) e Lantana camara var. nivea (Vent.) L.H.Bailey (coletada no Estado do Rio de Janeiro, mun. Cabo Frio), em estado fresco, por via oral, a vários bovinos. Doses únicas de 30 e $40 \mathrm{~g} / \mathrm{kg}$ ou doses repetidas de $10 \mathrm{~g} / \mathrm{kg}$, durante 4 a 5 dias, causaram sintomas graves com morte de parte dos animais em 15 dias; dose única de $20 \mathrm{~g} / \mathrm{kg}$ provocou sintomas moderados; doses de $5 \mathrm{~g} / \mathrm{kg}$ durante 22 dias produziram leves sintomas da intoxicação. As duas lantanas mostraram leve efeito acumulativo e toxicidade semelhante. Experimentos com L. camara var. aculeata (L.) Moldenke (coletada no Estado do Rio de Janeiro, mun. Vassouras e Itaguaí), nas mesmas dosagens, resultaram negativos.

Em outro estudo experimental, Riet-Correa et al. (1984) administraram Lantana glutinosa Poepp. (coletada em Santa Catarina), em estado fresco, por via oral, a bovinos, tendo provocado grave quadro de fotossensibilização e morte dos animais com doses únicas de 10, 20 e $40 \mathrm{~g} / \mathrm{kg}$.

Tokarnia et al. (1999) provocaram, em bovinos, pela administração de Lantana camara var. aculeata (L.) Moldenke (coletada no Estado do Rio de Janeiro, mun. Quatis), em estado fresco, por via oral, intoxicação grave com êxito letal, na dose única de $40 \mathrm{~g} / \mathrm{kg}$, intoxicação grave sem êxito letal na dose de $20 \mathrm{~g} / \mathrm{kg}$, leve intoxicação ou ausência de sintomas na dose de $10 \mathrm{~g} / \mathrm{kg}$ e ausência de sintomas na dose de $5 \mathrm{~g} / \mathrm{kg}$. Experimentos com doses repetidas permitiram concluir, que essa planta apresenta marcado efeito acumulativo quando ingerida em doses diárias de $1 / 4$ (10 g/ $/ \mathrm{kg})$ da dose letal; a administração de quatro doses diárias de $5 \mathrm{~g} / \mathrm{kg}$ (1/8 da dose letal) ou de oito doses de $2,5 \mathrm{~g} / \mathrm{kg}$ (1/16 da dose letal) reproduziram o quadro grave de intoxicação em parte dos animais, caracterizando ainda, o marcado efeito acumulativo da planta. Subdoses menores, de 1,25 g/kg (1/32 da dose letal), administradas durante 34 dias, não reproduziram quaisquer sinais clínicos.

Brito \& Tokarnia (1995) demonstraram, através de experimentos, que os ovinos têm a mesma sensibilidade que os bovinos, à intoxicação por Lantana camara var. aculeata (procedente do município de Quatis, Estado do Rio de Janeiro). Tanto a planta 
fresca, quanto a planta dessecada causaram doença grave com fotossensibilização e morte de todos os ovinos, na dosagem única de $40 \mathrm{~g} / \mathrm{kg}$ (planta fresca ou quantidade correspondente da planta dessecada); a dosagem de $10 \mathrm{~g} / \mathrm{kg}$ durante 4 dias seguidos também causou doença grave em quatro dos cinco animais; mas dois dos quatro animais severamente afetados se recuperaram.

Com o fim de esclarecer a diversidade de toxidez das lantanas na Austrália, Seawright (1965a) testou diversas amostras de L. camara (flores vermelhas, róseas, brancas e purpúreas) e uma amostra de L. montevidensis em 21 carneiros, nas doses únicas de 2 a $6 \mathrm{~g} / \mathrm{kg}$. Concluiu que a toxidez varia sobretudo de acordo com os taxa (fator genético) e, em menor grau, com a procedência da planta.
Este estudo teve como objetivo verificar, com maior abrangência, quais são as nossas lantanas tóxicas e qual o grau de toxidez destas últimas, através da experimentação em que amostras de lantanas, coletadas nas diversas regiões e áreas do Brasil, eram administradas sob forma dessecada a ovinos, com simultâneo rastreamento de históricos da ocorrência de fotossensibilização em bovinos e ovinos nos locais de coleta.

O uso de ovinos, como modelo experimental, e da planta dessecada é válido depois da conclusão de Brito \& Tokarnia (1995) demonstrando que bovinos e ovinos têm a mesma sensibilidade à toxidez das lantanas, e que a planta dessecada conserva a sua toxidez durante pelo menos um ano.

\section{Quadro 1. Experimentos com as folhas dessecadas de Lantana spp em ovinos}

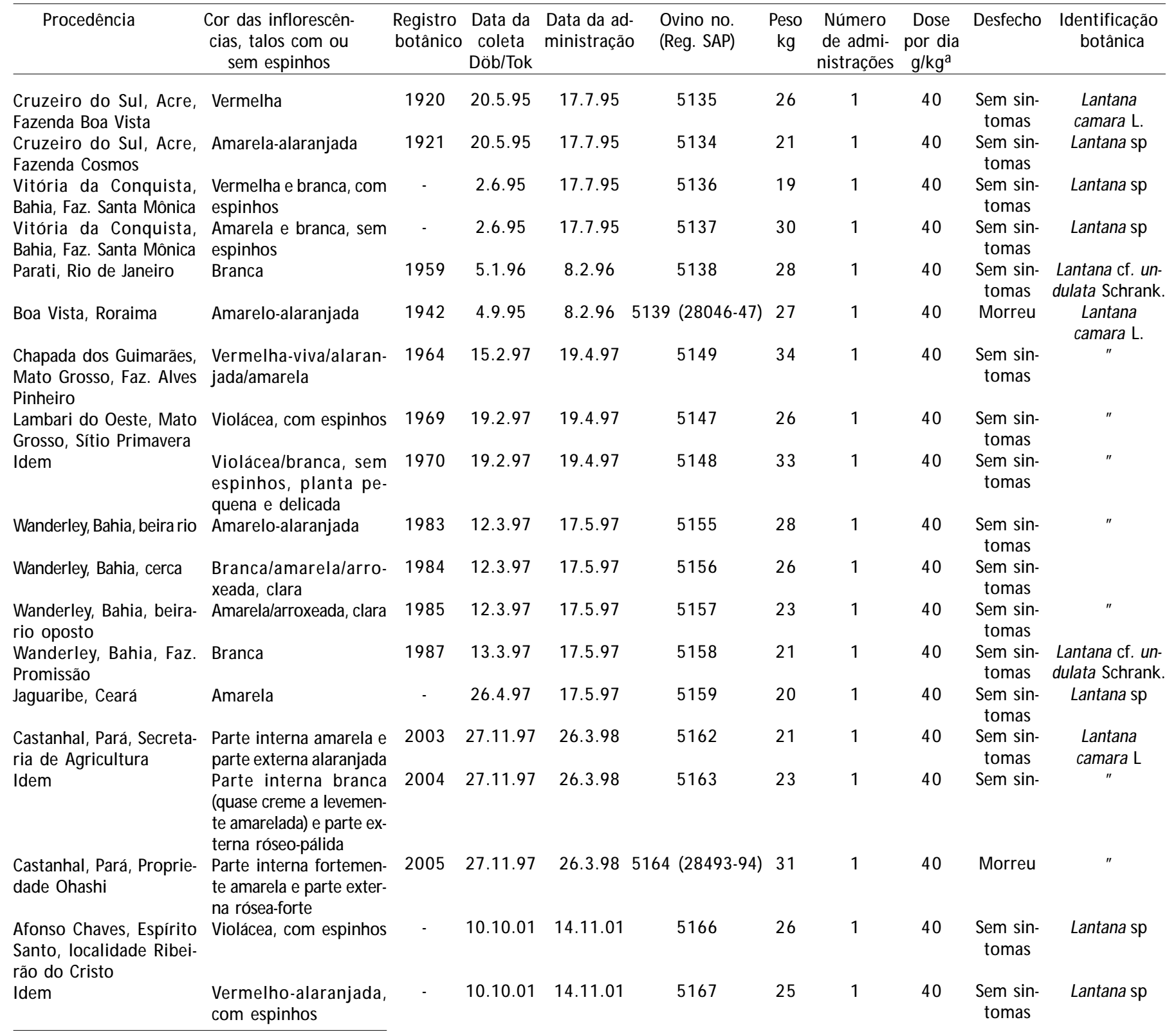

${ }^{a}$ As doses indicadas sempre correspondem às da planta fresca (Relação planta fresca:planta dessecada $=4: 1$ ). 
MATERIAL E MÉTODOS

Durante as nossas viagens de pesquisa, sempre que verificamos a ocorrência de lantanas em maiores quantidades em certa região, coletamos a planta fresca em quantidade suficiente para administrá-la a ovinos. Ao mesmo tempo fazíamos indagações sobre a ocorrência de surtos de fotossensibilização em bovinos e ovinos, e se esses animais foram trazidos de outros locais.

As amostras coletadas eram dessecadas à sombra e acondi- cionadas e transportadas e em sacos de pano. Sempre dentro de al guns meses após a coleta, estas amostras eram administradas por via oral a ovinos. As folhas eram levemente umedecidas e manualmente colocadas na boca dos animais, que então as mastigavam e deglutiam. Sempre eram administradas na dose única correspondente a $40 \mathrm{~g} / \mathrm{kg}$ da planta fresca (relação planta fresca: planta dessecada $=4: 1$ ). Cada amostra era dada a um ovino diferente, e cada animal era usado somente uma vez.

Quadro 2. Experimentos com as folhas frescas de Lantana spp em bovinos (Silva \& Couto 1971)

\begin{tabular}{|c|c|c|c|c|c|c|c|}
\hline Procedência & $\begin{array}{l}\text { Cor das inflo- } \\
\text { rescências }\end{array}$ & $\begin{array}{l}\text { Bovino no. } \\
\text { (Reg. SAP) }\end{array}$ & $\begin{array}{c}\text { Peso } \\
\mathrm{kg}\end{array}$ & $\begin{array}{l}\text { Número } \\
\text { de admi- } \\
\text { nistrações }\end{array}$ & $\begin{array}{c}\text { Dose } \\
\text { por dia } \\
\text { g/kg }\end{array}$ & Desfecho & $\begin{array}{l}\text { Identificação } \\
\text { botânica }\end{array}$ \\
\hline $\begin{array}{l}\text { Serra Talhada, } \\
\text { Pernambuco }\end{array}$ & Vermelha & 03 & 250 & 30 & 40 & $\begin{array}{l}\text { Adoeceu moderadamente, com meteorismo, ic- } \\
\text { terícia e leves sintomas de fotossensibilização }\end{array}$ & Lantana câmara L. \\
\hline Idem & Vermelha & 01 & 170 & 10 & 15 & Meteorismo, anorexia, constipação, diarréia & “ \\
\hline Idem & Vermelha & 02 & 250 & 10 & 15 & Anorexia, constipação, diarréia & “ \\
\hline Idem & Vermelha & 04 & 140 & 12 & 15 & Anorexia, constipação, diarréia & “ \\
\hline
\end{tabular}

Quadro 3. Experimentos com as folhas dessecadas de Lantana spp em bovinos (Silva \& Couto 1971)

\begin{tabular}{|c|c|c|c|c|c|c|c|}
\hline Procedência & $\begin{array}{l}\text { Cor das inflo- } \\
\text { rescências }\end{array}$ & $\begin{array}{l}\text { Bovino no. } \\
\text { (Reg. SAP) }\end{array}$ & $\begin{array}{l}\text { Peso } \\
\mathrm{kg}\end{array}$ & $\begin{array}{c}\text { Número } \\
\text { de admi- } \\
\text { nistrações }\end{array}$ & $\begin{array}{l}\text { Dose } \\
\text { por dia } \\
\mathrm{g} / \mathrm{kg}\end{array}$ & Desfecho & $\begin{array}{l}\text { Identificação } \\
\text { botânica }\end{array}$ \\
\hline $\begin{array}{l}\text { Serra Talhada, } \\
\text { Pernambuco }\end{array}$ & Vermelha & 05 & 260 & 30 & 40 & $\begin{array}{l}\text { Adoeceu moderadamente, com meteorismo, ic- } \\
\text { terícia e leves sintomas de fotossensibilização }\end{array}$ & Lantana câmara L. \\
\hline Idem & Vermelha & 06 & 156 & 30 & 20 & & $“$ \\
\hline Idem & Vermelha & 07 & 180 & 10 & 15 & Meteorismo, anorexia, constipação, diarréia & “ \\
\hline Idem & Vermelha & 08 & 200 & 10 & 15 & Anorexia, constipação, diarréia & $"$ \\
\hline
\end{tabular}

Quadro 4. Experimentos com as folhas frescas de Lantana spp em bovinos (Tokarnia et al. 1984)

\begin{tabular}{|c|c|c|c|c|c|c|c|c|c|c|}
\hline Procedência & $\begin{array}{l}\text { Cor das inflo- } \\
\text { rescências }\end{array}$ & $\begin{array}{l}\text { Registro } \\
\text { botânico } \\
\text { Döb/Tok }\end{array}$ & $\begin{array}{l}\text { Data da } \\
\text { coleta }\end{array}$ & $\begin{array}{l}\text { Data da ad- } \\
\text { ministração }\end{array}$ & $\begin{array}{l}\text { Bovino no. } \\
\text { (Reg. SAP) }\end{array}$ & $\begin{array}{l}\text { Peso } \\
\mathrm{kg}\end{array}$ & $\begin{array}{c}\text { Número } \\
\text { de admi- } \\
\text { nistrações }\end{array}$ & $\begin{array}{c}\text { Dose } \\
\text { por dia } \\
\mathrm{g} / \mathrm{kg}^{\mathrm{a}}\end{array}$ & Desfecho & $\begin{array}{l}\text { Identificação } \\
\text { botânica }\end{array}$ \\
\hline $\begin{array}{l}\text { Cáceres, Mato Grosso, Fa- } \\
\text { zenda N.S. Aparecida }\end{array}$ & Arroxeada & 1260 & 15.9 .76 & 16.9 .76 & $\begin{array}{c}3988 \\
(22177)\end{array}$ & 113 & 1 & 30 & Morreu & \multirow{2}{*}{$\begin{array}{c}\text { Lantana } \\
\text { tiliaefolia Cham. } \\
\text { " }\end{array}$} \\
\hline Idem & Arroxeada & $"$ & $"$ & $"$ & $\begin{array}{c}3593 \\
(22171)\end{array}$ & 102 & 4,5 & 10 & Morreu & \\
\hline $\begin{array}{l}\text { Cabo Frio, Rio de Janeiro, } \\
\text { Fazenda Vinhático }\end{array}$ & Branca & 1267 & 9.10 .76 & 10.10 .76 & $\begin{array}{c}3989 \\
(22227)\end{array}$ & 109 & 1 & 40 & $\begin{array}{l}\text { Adoeceu } \\
\text { gravemente }\end{array}$ & \multirow{3}{*}{$\begin{array}{c}\text { Lantana } \\
\text { nívea (Vent.) } \\
\text { camara var. } \\
\text { L.H. Bailey } \\
\text { " }\end{array}$} \\
\hline & & & & & & & & & & \\
\hline Idem & Branca & $"$ & $"$ & $"$ & $\begin{array}{c}3597 \\
(22295)\end{array}$ & 100 & 5 & 10 & $\begin{array}{c}\text { Adoeceu } \\
\text { gravemente }\end{array}$ & \\
\hline Idem & Branca & $"$ & $\begin{array}{l}9.10 .76 \\
\mathrm{e} \\
17.10 .76\end{array}$ & $"$ & 3994 & 89 & 22 & 5 & $\begin{array}{c}\text { Adoeceu } \\
\text { levemente }\end{array}$ & $"$ \\
\hline Idem & Branca & $"$ & 17.10 .76 & 19.10 .76 & 4121 & 101 & 1 & 10 & S.S & $"$ \\
\hline Idem & Branca & $"$ & $"$ & " & 4119 & 102 & 1 & 20 & $\begin{array}{l}\text { Adoeceu mo- } \\
\text { deradamente }\end{array}$ & $"$ \\
\hline Idem & Branca & $"$ & $"$ & 21.10 .76 & 4118 & 97 & 1 & 20 & $\begin{array}{l}\text { Adoeceu mo- } \\
\text { deradamente }\end{array}$ & $"$ \\
\hline $\begin{array}{l}\text { Vassouras, Rio de Janeiro, } \\
\text { Fazenda do Secretário }\end{array}$ & Amarelo-alaranjada & 1603 & 13.2 .79 & 13.2 .79 & 4198 & 120 & 1 & 58 & S.S. & $\begin{array}{l}\text { Lantana } \\
\text { camara var. } \\
\text { aculeata (L.) } \\
\text { Moldenke }\end{array}$ \\
\hline Idem & Amarelo-alaranjada & $"$ & $"$ & $"$ & 4199 & 162 & 1 & 40 & S.S. & $"$ \\
\hline Idem & Amarelo-alaranjada & $"$ & $"$ & $"$ & 4200 & 105 & 7 & 10 & S.S. & $"$ \\
\hline $\begin{array}{l}\text { Itaguaí, Rio de Janeiro, Sí- } \\
\text { tio Poranga }\end{array}$ & Amarelo-alaranjada & 1764 & 22.4 .81 & 22.4 .81 & 4355 & 111 & 4 & 10 & S.S. & $"$ \\
\hline Idem & Amarelo-alaranjada & $"$ & $"$ & $"$ & 4362 & 155 & 1 & 40 & S.S. & $"$ \\
\hline
\end{tabular}

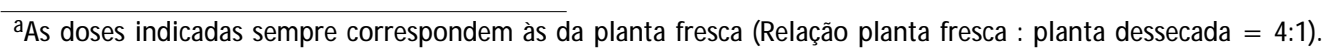


Os ovinos experimentais eram mantidos em compartimentos (boxes) individuais, com água à vontade e recebiam capimguatemala (Tripsacum laxum Nash.) picado no cocho, duas vezes por dia. Antes das administrações das amostras das lantanas, os animais ficavam em jejum durante 24 horas. Durante o dia fica- vam das 8 às 11 e das 14 às 17 horas num piquete ensolarado. Os animais antes e durante os experimentos eram examinados clinicamente com verificação da temperatura retal, determinação das frequências cardíaca e respiratória, verificação do funcionamento do rúmen, observação das mucosas visíveis, do com-

Quadro 5. Experimentos com as folhas frescas de Lantana spp em bovinos (Riet-Correa et al. 1984)

\begin{tabular}{|c|c|c|c|c|c|c|c|c|c|}
\hline Procedência & $\begin{array}{l}\text { Cor das inflo- } \\
\text { rescências }\end{array}$ & $\begin{array}{c}\text { Data da } \\
\text { coleta }\end{array}$ & $\begin{array}{c}\text { Data da } \\
\text { administração }\end{array}$ & $\begin{array}{c}\text { Bovino } \\
\text { no. }\end{array}$ & $\begin{array}{c}\text { Peso } \\
\mathrm{kg}\end{array}$ & $\begin{array}{c}\text { Número de } \\
\text { admninistrações }\end{array}$ & $\begin{array}{l}\text { Dose por } \\
\text { dia } \mathrm{g} / \mathrm{kg}\end{array}$ & Desfecho & $\begin{array}{c}\text { Identificação } \\
\text { botânica }\end{array}$ \\
\hline $\begin{array}{l}\text { Canoinha, San- } \\
\text { ta Catarina }\end{array}$ & Amarelo-alaranjada & $\begin{array}{l}\text { Janeiro } \\
\text { de } 1983\end{array}$ & $\begin{array}{l}\text { (Conservado em } \\
\text { congelador) }\end{array}$ & 7 & 68 a 121 & 1 & 40 & Morreu & $\begin{array}{l}\text { Lantana } \\
\text { glutinosa }\end{array}$ \\
\hline Idem & Amarelo-alaranjada & “ & “ & 8 & “ & 1 & 20 & $\begin{array}{l}\text { Poepp. } \\
\text { Morreu }\end{array}$ & “ \\
\hline Idem & Amarelo-alaranjada & “ & “ & 9 & “ & 1 & 10 & Morreu & “ \\
\hline Idem & Amarelo-alaranjada & $“$ & $“$ & 10 & $“$ & 1 & $\begin{array}{c}5 \\
\text { cretamente }\end{array}$ & Adoeceu dis- & $“$ \\
\hline
\end{tabular}

Quadro 6. Experimentos com as folhas frescas de Lantana spp em bovinos (Tokarnia et al. 1999)

\begin{tabular}{|c|c|c|c|c|c|c|c|c|c|c|}
\hline Procedência & $\begin{array}{l}\text { Cor das inflo- } \\
\text { rescências }\end{array}$ & $\begin{array}{l}\text { Registro } \\
\text { botânico } \\
\text { Döb/Tok }\end{array}$ & $\begin{array}{c}\text { Data da } \\
\text { coleta }\end{array}$ & $\begin{array}{l}\text { Data da pri- } \\
\text { meira ad- } \\
\text { ministração }\end{array}$ & $\begin{array}{r}\text { - Bovino no. } \\
\text { (Reg. SAP) }\end{array}$ & $\begin{array}{c}\text { Peso } \\
\mathrm{kg}\end{array}$ & $\begin{array}{l}\text { Número } \\
\text { de admi- } \\
\text { nistrações }\end{array}$ & $\begin{array}{l}\text { Dose } \\
\text { por dia } \\
\text { g/kg }\end{array}$ & Desfecho & $\begin{array}{c}\text { Identificação } \\
\text { botânica }\end{array}$ \\
\hline $\begin{array}{l}\text { Quatis, Rio de Janeiro, } \\
\text { Localidade de Falcão }\end{array}$ & Amarelo-alaranjada & 1904 & $\begin{array}{l}\text { Poucos dias } \\
\text { antes admi- } \\
\text { nistração } \\
\text { (geladeira) }\end{array}$ & 21.5 .92 & $\begin{array}{c}4928 \\
(25806-09)\end{array}$ & 127 & 4 & 10 & $\begin{array}{l}\text { Sacrificado } \\
\text { in extremis }\end{array}$ & \multirow[t]{2}{*}{$\begin{array}{c}\text { Lantana } \\
\text { camara var. } \\
\text { aculeata (L.) } \\
\text { Moldenke } \\
\text { " }\end{array}$} \\
\hline Idem & $"$ & $"$ & 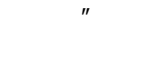 & $"$ & $\begin{array}{c}4923 \\
(25871-74)\end{array}$ & 176 & 1 & 40 & $\begin{array}{l}\text { Sacrificado } \\
\text { in extremis }\end{array}$ & \\
\hline Idem & $"$ & $"$ & $"$ & 24.8 .95 & 5229 & 142 & 5 & 5 & Adoeceu gravement & nte \\
\hline Idem & $"$ & $"$ & $"$ & $"$ & 5230 & 136 & 5 & 5 & Adoeceu gravement & nte \\
\hline Idem & $n$ & $"$ & $"$ & 18.9 .96 & 5273 & 190 & 1 & 5 & Sem sintomas & $"$ \\
\hline Idem & $"$ & $"$ & $"$ & " & 5270 & 197 & 1 & 10 & Adoeceu levemente & te \\
\hline Idem & $"$ & $"$ & “ & $"$ & 5528 & 272 & 1 & 20 & Adoeceu gravement & nte \\
\hline Idem & $"$ & $"$ & $“$ & 13.6.97 & 5249 & 162 & 9 & 2,5 & Adoeceu gravement & nte \\
\hline Idem & $"$ & $"$ & “ & $"$ & 5287 & 153 & 7 & 2,5 & Adoeceu gravement & nte \\
\hline Idem & $"$ & $"$ & “ & 14.5 .98 & 5292 & 138 & 34 & 1,25 & Sem sintomas & $“$ \\
\hline Idem & $"$ & $"$ & $“$ & & 5294 & 117 & 34 & 1,25 & Sem sintomas & $“$ \\
\hline Idem & $"$ & $"$ & “ & 25.6 .98 & 5296 & 142 & 19 & 2,5 & Adoeceu gravement & nte \\
\hline Idem & $"$ & $"$ & “ & $"$ & 5297 & 135 & 32 & 2,5 & Sem sintomas & “ \\
\hline Idem & $"$ & $"$ & “ & 14.7 .98 & 5292 & 151 & 1 & 20 & Adoeceu gravement & nte \\
\hline Idem & $"$ & $"$ & $“$ & 24.7 .98 & 5294 & 119 & 1 & 10 & Sem sintomas & $“$ \\
\hline
\end{tabular}

Quadro 7. Experimentos com as folhas frescas de Lantana spp em ovinos (Brito \& Tokarnia 1995)

\begin{tabular}{|c|c|c|c|c|c|c|c|c|c|c|}
\hline Procedência & $\begin{array}{l}\text { Cor das inflo- } \\
\text { rescências }\end{array}$ & $\begin{array}{l}\text { Registro } \\
\text { botânico } \\
\text { Döb/Tok }\end{array}$ & $\begin{array}{l}\text { Data da } \\
\text { coleta }\end{array}$ & $\begin{array}{l}\text { Data da ad- } \\
\text { ministração }\end{array}$ & $\begin{array}{l}\text { Ovino no. } \\
\text { (Reg. SAP) }\end{array}$ & $\begin{array}{c}\text { Peso } \\
\mathrm{kg}\end{array}$ & $\begin{array}{c}\text { Número } \\
\text { de admi- } \\
\text { nistrações }\end{array}$ & $\begin{array}{l}\text { Dose } \\
\text { por dia } \\
\text { g/kg }\end{array}$ & Desfecho & $\begin{array}{c}\text { Identificação } \\
\text { botânica }\end{array}$ \\
\hline $\begin{array}{l}\text { Quatis, Rio de Janei- } \\
\text { ro, Localidade de Fal- } \\
\text { cão }\end{array}$ & Amarelo-alaranjada & 1904 & 16.8 .93 & 17.8 .93 & $\begin{array}{c}5087 \\
(26824-828)\end{array}$ & 12,5 & 1 & 40 & Morreu & $\begin{array}{c}\text { Lanatana } \\
\text { câmara var. } \\
\text { aculeata (L.) } \\
\text { Moldenke }\end{array}$ \\
\hline Idem & $“$ & $“$ & 26.7 .93 & 27.7 .93 & $\begin{array}{c}5092 \\
(26700-704)\end{array}$ & 26,5 & 1 & 40 & Morreu & \\
\hline Idem & $“$ & $“$ & 26.7 .93 & 27.30 .7 .93 & 5093 & 26 & 4 & 10 & Adoeceu gravemente & $“$ \\
\hline Idem & " & $“$ & 16.7 .93 & $17-20.7 .93$ & $\begin{array}{c}5094 \\
(26838-842)\end{array}$ & 41,5 & 4 & 10 & Morreu & " \\
\hline Idem & $“$ & $“$ & 12.6 .97 & $\begin{array}{c}21.6 .97 \\
\text { (geladeira) }\end{array}$ & 5160 & 17 & 1 & 20 & $\begin{array}{l}\text { Adoeceu moderada- } \\
\text { mente; não deixa- } \\
\text { mos mais no sol }\end{array}$ & 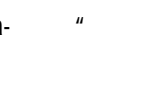 \\
\hline Idem & “ & $“$ & 12.6 .97 & $\begin{array}{l}21.6 .97 \\
\text { (geladeira) }\end{array}$ & 5161 & 14 & 1 & 20 & $\begin{array}{l}\text { Adoeceu gravemente } \\
\text { se deixasse no sol } \\
\text { ia morrer }\end{array}$ & te; \\
\hline
\end{tabular}


Quadro 8. Experimentos com as folhas dessecadas de Lantana spp em ovinos (Brito \& Tokarnia 1995)

\begin{tabular}{|c|c|c|c|c|c|c|c|c|c|c|}
\hline Procedência & $\begin{array}{l}\text { Cor das inflo- } \\
\text { rescências }\end{array}$ & $\begin{array}{l}\text { Registro } \\
\text { botânico } \\
\text { Döb/Tok }\end{array}$ & $\begin{array}{l}\text { Data da } \\
\text { coleta }\end{array}$ & $\begin{array}{l}\text { Data da ad- } \\
\text { ministração }\end{array}$ & $\begin{array}{l}\text { Ovino no. } \\
\text { (Reg. SAP) }\end{array}$ & $\begin{array}{l}\text { Peso } \\
\mathrm{kg}\end{array}$ & $\begin{array}{l}\text { Número } \\
\text { de admi- } \\
\text { nistrações }\end{array}$ & $\begin{array}{l}\text { Dose } \\
\text { por dia } \\
\mathrm{g} / \mathrm{kg}\end{array}$ & Desfecho & $\begin{array}{c}\text { Identificação } \\
\text { botânica }\end{array}$ \\
\hline $\begin{array}{l}\text { Quatis, Rio de Janei- } \\
\text { ro, Localidade de Fal- } \\
\text { cão }\end{array}$ & Amarelo-alaranjada & 1904 & $\begin{array}{l}26.7 .93 \\
\text { e } 18.8 .93\end{array}$ & 27 a 30.10 .93 & 5112 & 30 & 4 & 10 & Adoeceu levemente & $\begin{array}{l}\text { Lantana } \\
\text { camara var. } \\
\text { aculeata (L.) } \\
\text { Moldenke }\end{array}$ \\
\hline Idem & $"$ & $"$ & $"$ & 30.10 .93 & $\begin{array}{c}5122 \\
(27209-213)\end{array}$ & 29.5 & 1 & 40 & Morreu & \\
\hline Idem & $"$ & $"$ & $"$ & 1.2 .94 & $\begin{array}{c}5126 \\
(27327-330)\end{array}$ & 25 & 1 & 40 & Morreu & $"$ \\
\hline Idem & $"$ & $"$ & $"$ & 1 a 4.2 .94 & 5125 & 25 & 4 & 10 & Adoeceu levemente & $"$ \\
\hline Idem & $"$ & $"$ & $"$ & 3.8 .94 & $\begin{array}{c}5130 \\
(27500-503)\end{array}$ & 22 & 1 & 40 & Morreu & $"$ \\
\hline Idem & $"$ & $"$ & $"$ & 3 а 6.8 .94 & $\begin{array}{c}5131 \\
(27504-508)\end{array}$ & 18.5 & 4 & 10 & Morreu & $"$ \\
\hline
\end{tabular}

portamento, do consumo de água e de capim, do aspecto das fezes e da urina e, sobretudo, do aparecimento de sinais de fotossensibilização. Nos casos de morte, procedia-se imediatamente a necropsia, complementada por exames histopatológicos.

\section{RESULTADOS}

No Quadro 1 constam os principais dados sobre os experimentos realizados.

\section{DISCUSSÃO E CONCLUSÕES}

Para o estudo comparativo da toxidez das lantanas para bovinos e ovinos podemos aproveitar com a mesma equivalência todos os experimentos realizados no Brasil nessas duas espécies animais e com a planta fresca ou dessecada, pois conforme ficou demonstrado (Brito \& Tokarnia 1995), a toxidez dessas plantas é igual para bovinos e ovinos e a planta não perde em toxidez pela dessecagem, além de mantê-la pelo menos durante um ano. Para facilitar este estudo comparativo colocamos em seguida os dados publicados anteriormente também em Quadros dispostos da mesma maneira que os apresentados nos experimentos em ovinos com as lantanas dessecadas.

Pela leitura dos Quadros 1 a 8, confirma-se o que foi visto na Austrália, que nem todas as espécies de Lantana e nem todos os taxa de Lantana camara são tóxicos. Verifica-se, que foram tóxicas as amostras de lantanas coletadas em Boa Vista (RO), Castanhal (PA), Cáceres (MT), Serra Talhada (PE), Cabo Frio e Quatis (RJ) e Canoinhas (SC). No entanto, outras amostras, procedentes de Cruzeiro do Sul (Acre), Castanhal (PA), Chapada dos Guimarãese Lambari d'Oeste (MT), Jaguaribe (CE), Vitória da Conquista e Wanderley (BA), Vitória (ES), Vassouras, Parati e Itaguaí (RJ), não revelaram toxidez nas doses administradas, de $40 \mathrm{~g} / \mathrm{kg}$.

A dose letal das amostras submetidas à experimentação no Brasil foi bastante constante ( $40 \mathrm{~g} / \mathrm{kg}$ ), com duas exceções: as folhas frescas da lantana procedente de Canoinha (SC) foram muito mais tóxicas $(10 \mathrm{~g} / \mathrm{kg}$ ) e as folhas frescas e as dessecadas da lantana de Serra Talhada (PE) foram muito menos tóxicas, pois somente causaram intoxicação não-letal com a dose de $40 \mathrm{~g} /$ kg/dia administradas durante 30 dias. Históricos sobre a obser- vação de fotossensibilização em bovinos, ligados à ingestão de lantanas foram obtidos em Serra Talhada (PE), Cáceres (MT), Cabo Frio (RJ), Quatis (RJ) e Canoinhas (SC).

Através da leitura dos Quadros verifica-se que não é possível estabelecer uma correlação entre a cor das inflorescências das lantanas e sua toxidez, confirmando a constatação de Seawright (1963), que a capacidade de intoxicar não está necessariamente relacionada com a cor das flores.

Agradecimentos.- A determinação botânica da maior parte das lantanas foi realizada pelo Prof. Pedro Germano Filho, do Departamento de Botânica, Universidade Federal Rural do Rio de Janeiro.

\section{REFERÊNCIAS}

Aguilera R., Zaldivar V., Margolles E., Bulnes C. \& Fuentes O. 1986. Indicadores del funcionamento hepatico en la intoxicacion experimental aguda de vacas por Lantana camara. Revta Salud Animal, Habana, 8(3):211220.

Aluja A.S. 1970. Lantana camara poisoning in cattle in Mexico. Vet. Rec. 86:628.

Aluja A.S. 1971. Further investigation regarding the toxicity of members of the genus Lantana in Mexico. Anais XIX Congr. Mundial Veterinaria, Mexico, v.1, p.327-331.

Brito M.F. 1995. Sensibilidade do coelho à intoxicação por Lantana camara var. aculeata (Verbenaceae) em estado fresco e dessecado. Pesq. Vet. Bras. 15(4):107-110.

Brito M.F. \& Tokarnia C.H. 1995. Estudo comparativo da toxidez de Lantana camara var. aculeata em bovinos e ovinos. Pesq. Vet. Bras. 15(2/3):79-84.

Brooks O.H. 1961. Lantana poisoning. Qd Agric. J. 87:641-642.

Furie N., Van der Lugt J.J., Newsholme S.J. \& Nel P.W. 1987. Acute Lantana camara toxicity in cattle. J. S. Afr. Vet. Assoc. 58(4):173-178.

Gobinath C. \& Ford E.J.H. 1969. The effect of Lantana camara on the liver of sheep. J. Pathol. 99(1):75-85.

Hall W.T.K. 1964. Plant toxicosis of tropical Australia. Aust. Vet. J. 40:176182

Harley K.L.S. 1973. Biological control of Lantana in Australia. Proc. 3rd Int. Symp. Biol. Control Weeds, Montpellier, France, p.23-29.

Kalra D.S., Dixit S.N., Verma P.C. \& Dwivedi P. 1984. Studies on experimental Lantana poisoning in buffalo calves with special reference to its pathology and histopathology. Haryana Vet. 23(2):98-105.

Láu H.D. 1990. Efeitos tóxicos de Lantana camara e de Pithomyces chartarum em búfalos. Doc. 54, Embrapa-CPATU, Belém, Pará. 18p. 
Obwolo M.J., Odiawo G.O. \& Goedegebuure S.A. 1990. Clinicopathological features of experimental low-dose Lantana camara poisoning in indigenous Zimbabwean goats. Zimbabwe Vet. J. 21(1):1-7.

Obwolo M.J., Basudde C.D.K., Odiawo G.O. \& Goedegebuure S.A. 1991. Clinicopathological features of experimental acute Lantana camara poisoning in indigenous Zimbabwean goats. Bull. Anim. HIth Prod. in Africa 39(3):339-346.

Pass M.A. 1986. Current ideas on the pathophysiology and treatment of lantana poisoning of ruminants. Aust. Vet. J. 63(6):169-171.

Pass M.A., Gemmell R.T. \& Heath T.J. 1978. Effect of Lantana on the ultrastructure of the liver of sheep. Toxicol. Appl. Pharmacol. 43:589596.

Riet-Correa F., Méndez M.C., Schild A.L., Riet-Correa I. \& Silva Neto S.R. 1984. Intoxicação por Lantana glutinosa em bovinos no Estado de Santa Catarina. Pesq. Vet. Bras. 4:147-153.

Sanders D.A. 1946. Lantana poisoning in cattle. J. Am. Vet. Med. Assoc. 89(833):139-141.

Seawright A.A. 1963. Studies on experimental intoxication of sheep with Lantana camara. Aust. Vet. J. 39:340-344.

Seawright A.A. 1964. Studies on the pathology of experimental lantana (Lantana camara L.) poisoning of sheep. Pathol. Vet. 1:504-529.

Seawright A.A. 1965a. A possible mechanism of intrahepatic obstruction in lantana poisoning. Aust.Vet. J. 41:116-119.

Seawright A.A. 1965b. Toxicity of Lantana spp. in Queensland. Aust. Vet. J. $41: 235-238$

Seawright A.A. 1965c. Electron microscopic observations of the hepatocytes of sheep in lantana poisoning. Pathol. Vet. 2:175-196.
Seawright A.A. 1965d. Toxicity for the guinea pig of an extract of Lantana camara. J. Comp. Pathol. 75:215-221.

Seawright A.A. \& Allen J.G. 1972. Pathology of the liver and kidney in lantana poisoning of cattle. Aust.Vet. J. 48:323-331.

Sharma O.P., Dawra R.K., Krishna L. \& Makkar H.P.S. 1988. Toxicity of lantana (Lantana camara L.) leaves and isolated toxins to rabbits. Vet. Hum. Toxicol. 30(3):214-218.

Silva F.M. \& Couto E.S. 1971. Intoxicação experimental de bovinos pela Lantana camara no Estado de Pernambuco. Arqs Esc. Vet., Univ. Fed. Minas Gerais, 23:77-89.

Steyn D.G. \& Van der Walt S.J. 1941. Recent investigations into the toxicity of known and unknown poisonous plants in the Union of South Africa, XI. Onderstepoort J. Vet. Sci. Anim. Ind. 16(1,2):121-147.

Tokarnia C.H., Döbereiner J., Lazzari A.A. \& Peixoto P.V. 1984. Intoxicação por Lantana spp (Verbenaceae) em bovinos nos Estados de Mato Grosso e Rio de Janeiro. Pesq. Vet. Bras. 4(4):129-141.

Tokarnia C.H., Armién A.G., Barros S.S., Peixoto P.V. \& Döbereiner J. 1999. Estudos complementares sobre a toxidez de Lantana camara (Verbenaceae) em bovines. Pesq. Vet. Bras. 19(3/4):128-132.

Turbet C.R. 1928. Dermatitis in cattle in Fiji. Agric. J., Fiji, 1:46-53.

Turbet C.R. 1931. Lantana poisoning of cattle in Fiji. Agric. J., Fiji, 4:2429.

Yadava J.N.S. \& Verma N.S. 1978. An outbreak of lantana poisoning in domesticated animals. Indian Vet. Med. J. 2:1-9.

Youssef M.S., Mahmoud A.Z. \& Hafez A.M. 1984. Pathological studies on the experimental intoxication of rabbits with Lantana camara. Assiut Vet. Med. J. 12(24):127-132. 